\title{
Modelo de seguimiento a proyectos como herramienta en la interventoría de contratos
}

\author{
César Augusto Prieto Cárdenas ${ }^{1}$ \\ Universidad Nacional de Colombia \\ caprietoc@unal.edu.co
}

\section{Fecha de recepción: 08 de mayo de 2018}

Fecha de aprobación: 22 de julio de 2018

DOI: https://doi.org/10.21158/01208160.n0.2018.2020

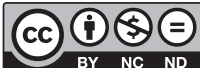

Cómo citar este artículo / To reference this article / Comment citer cet article / Para citar este artigo:

Prieto Cárdenas, C. A. (2018). Modelo de seguimiento a proyectos como herramienta en la interventoría de contratos. Revista EAN, Edición especial, pp 109-123.DOI: https://doi.org/10.21158/01208160.n0.2018.2020

\section{Resumen}

El problema más común que enfrentan las administraciones públicas, es la ausencia de un método eficaz y sistemático para realizar el seguimiento a través de mediciones de avance de la gestión y evaluar los resultados de aquellas iniciativas de inversión que están orientadas a financiar de manera directa y de forma recurrente la operación de las entidades. En este contexto, el presente trabajo pretende desarrollar un método cuantitativo que permita realizar la medición del logro del objeto contractual a través del avance de las obligaciones del contratista acordadas para la entrega del bien o servicio. La propuesta metodológica que se presenta a continuación busca implementar el Modelo de Seguimiento a la ejecución del avance del objeto contractual (Mosac) en sus dos niveles: estratégico — componentes - y operativo —obligaciones—. En este sentido, el Modelo de Seguimiento MOSAC se convierte en un instrumento de implementación a la ejecución efectiva de los contratos que lleve a cabo la Administración pública.

\section{Palabras Clave}

Control de proyectos, administración pública, evaluación de proyectos, ejecución de proyectos, revisión de proyectos.

\footnotetext{
${ }^{1}$ Administrador de empresas Universidad Nacional de Colombia. Magister en Ingeniería Industrial, Universidad Nacional de Colombia. Especialista en Evaluación Social de Proyectos, Universidad de los Andes. Estructurador de Proyectos en Metodología de Marco Lógico, Banco Interamericano de Desarrollo.
} 


\section{Project follow- up model as a tool in contract auditing}

Abstract. The most common problem found in public administration is the lack of an efficient and systematic method to follow up the development of projects through the advancement management measurements and the evaluation of investment outcomes which are oriented to finance the operation of companies in a direct and systematic way. In this context, this article aims at describing a quantitative method which allows the measurement of the contract object achievement based on the advancement of obligations of the contractor to guarantee the adequate delivery of a product or service. This proposed methodology tries to implement a Follow-up Method for the measurement of the contractual object execution (Mosac) at its two levels. Strategic - components - and operative - obligations-. In this respect, the Follow-up Model MOSAC becomes an implementation tool in the effective execution of contracts carried out by public administration.

key words: Project management control, public administration, project evaluation, project execution, project follow-up.

\section{$\longrightarrow$ \\ Modèle de suivi de projets devenant un outil d'audit de contrat}

Résumé. Le problème le plus commun auquel sont confrontées les administrations publiques se trouve être le manque de méthode efficiente et systématique permettant le suivi des progrès de la gestion et l'évaluation des résultats des investissements visant à financer directement et de manière récurrente le fonctionnement des entités. Dans ce contexte, ce document vise à mettre en place une méthode quantitative permettant de mesurer l'avancement de la réalisation du contrat et les obligations de l'entrepreneur ayant accepté de livrer le bien ou le service. La proposition méthodologique présentée ci-après vise à la mise en cuvre du modèle de suivi et de l'objet contractuel-Msoc-sur deux niveaux: - stratégique —composants - et opérationnel —obligations-. En ce sens, le modèle de suivi MSOC devient un instrument de mise en cuvre pour l'exécution efficace des contrats exécutés par l'administration publique.

Mots clefs: Contrôle de projets, administration publique, évaluation de projets, exécution de projets, revue de projets.

\section{Modelo de acompanhamento a projetos como ferramenta na interventoria de contratos}

Resumo. O problema mais comum que enfrentam as administrações públicas, é a ausência de um método eficaz e sistemático para realizar o acompanhamento através de medidas de avanço do gerenciamento e avaliar os resultados daquelas iniciativas de investimento que estão orientadas a financiar de maneira direta e de forma frequente a operação das entidades. Neste contexto, o presente trabalho pretende desenvolver um método quantitativo que permita realizar a medida do lucro do objeto contratual através do avanço das obrigações da contratante acordadas para a entrega do bem ou do serviço. A proposta metodológica que se apresenta a seguir procura implementar o Modelo de Acompanhamento da execução do avanço do objeto contratual (Mosac) em seus dois níveis: estratégico - componentes - e operativo - obrigações - Neste sentido, o Modelo de Acompanhamento MOSAC se torna um instrumento de implementação da execução efetiva dos contratos que realize a administração pública.

Palavras-chave: Controle de projetos, administração pública, avaliação de projetos, execução de projetos, revisão de projetos. 


\section{Marco conceptual}

$\mathrm{E}^{n}$ n términos generales, se entiende que un contrato estatal no constituye un fin en sí mismo, sino que ante todo debe entenderse como un instrumento mediante el cual se vale la Administración para cumplir con los cometidos estatales contemplados explícitamente en el preámbulo y en el artículo 2 de la Constitución Política. Así mismo, estos cometidos se podrían integrar de manera general en la concepción clásica del servicio público, al estar esencialmente vinculados a la satisfacción de las necesidades colectivas y a la preeminencia del interés general (Balcázar, 2012).

Por esta razón, el Estatuto General de Contratación de la Administración Pública advierte que los contratos estatales responden a una necesidad comunitaria. De ahí que, al celebrarlos, sea una obligación ineludible para la Administración garantizar la calidad de los bienes y de los servicios que contrata.

En otras palabras, las entidades estatales tienen la obligación de asegurar el cumplimiento del objeto contractual de los contratos que celebren, para lo cual tendrán la dirección general y responsabilidad de ejercer el control y la vigilancia de la ejecución del contrato (Ley 80/1993, de 28 de octubre, art. 14, num. 1). Como manifestación de este deber, se encuentran las figuras de la supervisión e interventoría (Colombia Compra Eficiente, 2016). Según la Ley 1474/2011, de 12 de julio, la supervisión de un contrato estatal consiste en «el seguimiento técnico, administrativo, financiero, contable y jurídico que sobre el cumplimiento del objeto del contrato, es ejercido por la misma entidad estatal cuando no se requieren conocimientos especializados» (art. 83). En esta misma ley, se define la interventoría de un contrato estatal como el seguimiento técnico que sobre el cumplimiento del contrato realice una persona natural o jurídica contratada para tal fin por la Entidad Estatal, cuando el seguimiento del contrato suponga conocimiento especializado en la materia, o cuando la complejidad o la extensión del mismo lo justifiquen. No obstante, lo anterior cuando la entidad lo encuentre justificado y acorde a la naturaleza del contrato principal, podrá contratar el seguimiento administrativo, técnico, financiero, contable, jurídico del objeto o contrato dentro de la interventoría. (art. 83, par. 3)

En este orden de ideas, tanto los supervisores como los interventores necesitan herramientas cuantitativas que les permitan de manera objetiva medir el avance en la ejecución del objeto contractual.

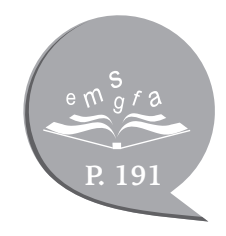




\section{Modelo de seguimiento al avance contractual}

$\mathbf{Z}^{1}$ modelo de seguimiento al avance Econtractual (Mosac) que se propone surge como respuesta a los supervisores e interventores que necesitan medir el avance de las acciones que ejecutan los contratistas. De esta forma, se cuenta con una herramienta cuantitativa que les permita verificar el cumplimiento de la calidad, la cantidad, el cronograma y los costos en la ejecución del objeto del contrato.

El diseño del Mosac está soportado en dos herramientas metodológicas: los componentes del contrato - nivel estratégico-y las obligaciones específicas - nivel operativo-. La figura 1 muestra cómo se podría explicar.

Figura 1. Modelo de seguimiento al avance contractual

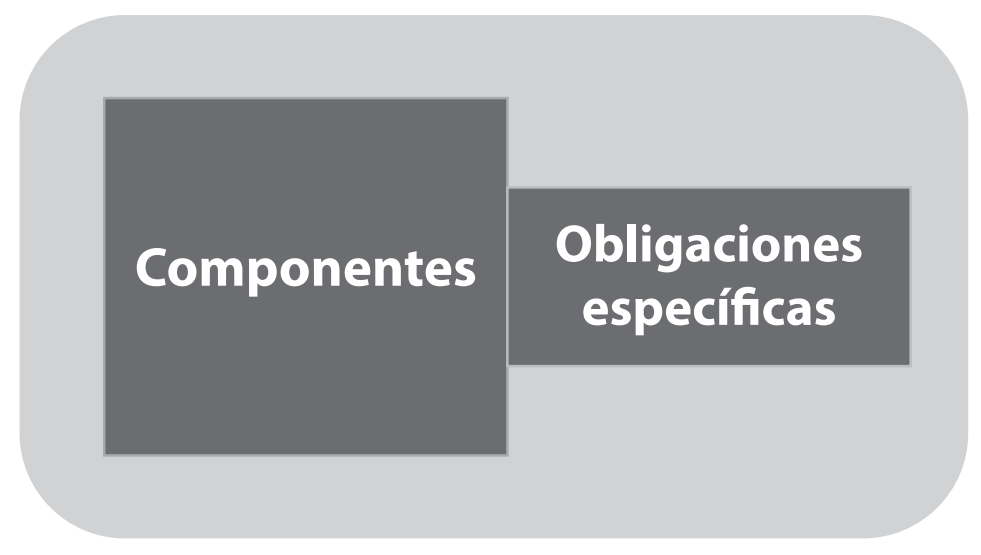

Fuente. Elaboración propia.

\subsection{Ruta crítica Mosac}

La ruta crítica que sigue el Mosac para apoyar el proceso de cuantificación de avance que permite demostrar el grado de contribución que hace al logro del objeto contractual se muestra en la figura 2. 
Figura 2. Ruta crítica Mosac

Fuente. Elaboración propia.

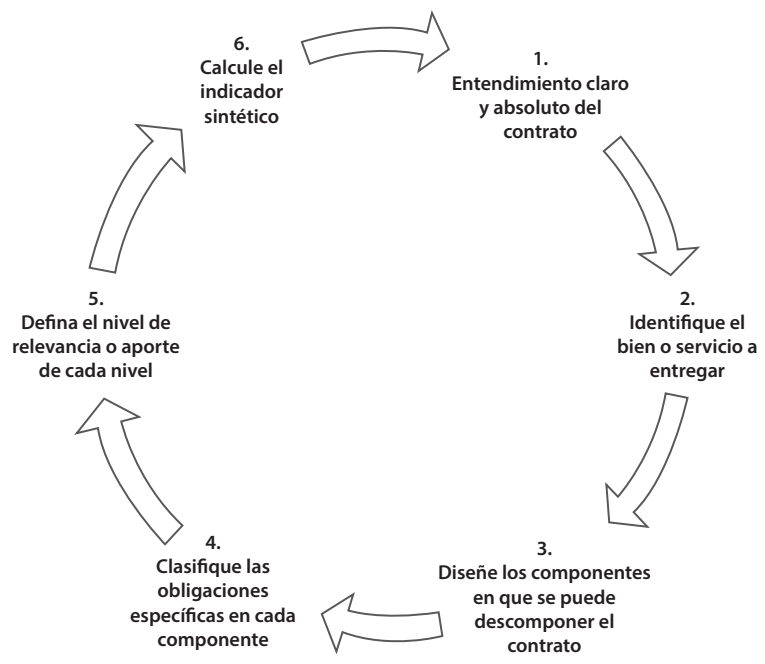

\subsubsection{Entendimiento claro y absoluto del contrato}

Al respecto, es muy importante conocer en detalle los documentos que dieron origen a la relación contractual, es decir, los términos de referencia, la propuesta y el contrato. Se debe llegar a comprender lo que espera el contratante a lo largo de la ejecución del contrato.

En este sentido, es esencial que una vez se reciba el encargo de seguimiento, se estudien de manera detallada los términos de referencia en los que se identifiquen entregables que no necesariamente quedan siempre incluidos en el contrato. Así, se sugiere realizar un listado de todas aquellas acciones y productos que el contratante está solicitando sean realizados por el contratista. Listado que posteriormente se compara con los entregables que quedaron finalmente contenidos en el contrato.

En este contexto, es necesario resaltar la naturaleza jurídica que el pliego de condiciones ostenta. En efecto, como bien lo ha establecido el Concepto 1642/2005, de 9 de junio, «el pliego de condiciones o términos de referencia han sido definidos por la doctrina y la jurisprudencia como la ley del contrato, indicando con ello su intangibilidad y obligatoriedad», hasta tal punto que la minuta del contrato no puede modificar las estipulaciones contractuales contenidas en el pliego de condiciones ${ }^{2}$.

\footnotetext{
2 La jurisprudencia ha manifestado que en el pliego de condiciones se «constituye no solo la ley del proceso de selección, por establecer todas las normas de participación, evaluación y adjudicación de la respectiva licitación o concurso, sino también la ley del futuro contrato, en la medida en que debe establecer el contenido del negocio jurídico que se celebrará como resultado de la licitación y especificar los derechos y obligaciones que surgirán para las partes del contrato a adjudicar. Así lo ha considerado la jurisprudencia de la corporación, para la cual una vez suscrito el contrato, las disposiciones del pliego hacen parte integral de su clausulado y se imponen sobre las pactadas en el contrato mismo» (Sentencia 15666/2011, de 10 de marzo).
} 
La comparación entre el pliego y el contrato es importante, puesto que es necesario realizar un cruce entre las obligaciones contractuales y las acciones que permiten llevar a cabo los entregables, con el fin de garantizar los principios de responsabilidad contenidos en el artículo 26 de la Ley 80/1993, de 28 de octubre:

- Respetar los fines de la contratación;

- Vigilar la correcta ejecución de lo pactado; $y$

- Velar por la protección de los derechos de la entidad y del contratista.

\subsubsection{Identificación del bien o servicio por entregar}

Debe tenerse en cuenta que el Estado contrata con un tercero la generación de productos - bienes o servicios- que deben entregarse en un tiempo determinado cronograma- con unas especificaciones establecidas — calidad - a un precio definido - costo- y en un número de unidades acordadas — cantidad-.

Conceptualmente, se entiende un bien como un entregable tangible y un servicio como un entregable intangible. Lo que es relevante tener en cuenta es que sea bien o servicio debe ser entregable, de tal forma que la entidad contratante pueda recibirlo y ser específico, es decir, que esté claramente identificado.

Como ejemplo podría mencionarse que «zapatos» o "comida» no son bienes, puesto que, a pesar de ser tangibles, no cumplen la característica de específicos. Diferente sería «tenis blancos de tela" o "papa criolla», que están especificados.

\subsubsection{Diseño de componentes del contrato}

Para alcanzar los fines de la contratación, es necesario identificar los factores críticos de éxito (Baccarini y Collins, 2003). En nuestro caso, es indispensable identificar los componentes en los que podrían agruparse las distintas obligaciones específicas, que deben cumplirse y que garantizan el logro del objeto contractual.

\subsection{Caso práctico}

Como resultado de los análisis que durante los últimos años el autor ha tenido que realizar como director de proyectos de interventoría y con el ánimo de aplicar de manera pedagógica la implementación de los conceptos que aquí se exponen, se propone un ejemplo de un contrato suscrito entre una entidad estatal y un contratista.

El primer paso dentro del Mosac consiste en hacer una selección de los componentes que se van a tomar en consideración. Su selección se debe hacer de acuerdo con los criterios formales que el contratante considera son los requeridos para la ejecución del contrato.

Para realizar la selección de componentes, es necesario comprender totalmente los fines de la contratación que pretende la entidad del Estado - bienes y servicios- e identificar las fases - componentes- necesarias para el logro del objeto contractual. Es relevante mencionar que las fases que se definan deben permitir que las obligaciones específicas puedan posteriormente ser clasificadas en cada una de ellas. Es decir, no podrá existir un componente sin obligaciones $\mathrm{u}$ obligaciones sin componente. 
A continuación, se presenta una aplicación práctica sobre un conjunto de datos referidos al análisis de avance de un contrato suscrito entre una entidad del Estado y un contratista. Los componentes se definieron en la tabla 1.

Tabla 1. Componentes

\begin{tabular}{|c|}
\hline Componentes \\
\hline Administrativo \\
\hline Mantenimiento \\
\hline Desplazamiento \\
\hline Seguridad \\
\hline Cumplimiento normas \\
\hline
\end{tabular}

Fuente. Elaboración propia.

\subsubsection{Clasificar las obligaciones específicas}

De acuerdo con los componentes, agrupar las obligaciones garantiza que exista un seguimiento integral del avance del contrato, lo cual permite tener una visión holística de la ejecución.
Deberá tenerse en cuenta que existen dos tipos de obligaciones específicas: las misionales y las funcionales (Tabla 2).

Tabla 2. Tipos de obligaciones

\begin{tabular}{|c|l|}
\hline & $\begin{array}{l}\text { Son aquellas directamente } \\
\text { relacionadas con la producción } \\
\text { de los bienes o servicios objeto } \\
\text { de la contratación, es decir, } \\
\text { las que son esenciales, sin } \\
\text { misionales } \\
\text { cuya ejecución se afectaría la } \\
\text { producción de los bienes o } \\
\text { servicios. }\end{array}$ \\
\hline \multirow{2}{\text{Obligaciones}}{$\begin{array}{l}\text { Son aquellas necesarias para } \\
\text { funcionales }\end{array}$} & $\begin{array}{l}\text { llevar a cabo la operación, en } \\
\text { general, son de apoyo para } \\
\text { el normal funcionamiento } \\
\text { del contrato. Entre ellas, } \\
\text { encontramos la firma de actas, } \\
\text { constituir pólizas, hacer los } \\
\text { pagos de aportes parafiscales, } \\
\text { etc. }\end{array}$ \\
\hline
\end{tabular}

Fuente. Elaboración propia.

Una vez que se seleccionaron los componentes, el segundo paso consiste en realizar una clasificación de cada una de las obligaciones del contrato en cada uno de ellos, que una vez revisadas por la mesa técnica dieron como resultado la tabla 3 .

Tabla 3. Clasificación obligaciones en los componentes

\begin{tabular}{|c|c|c|c|c|}
\hline $\begin{array}{l}\text { Componente } \\
\text { funcional }\end{array}$ & & \multicolumn{2}{|c|}{$\begin{array}{l}\text { Componentes } \\
\text { misionales }\end{array}$} & \\
\hline ADMINISTRATIVO & MANTENIMIENTO & DESPLAZAMIENTO & SEGURIDAD & CUMPLIMIENTO DE NORMAS \\
\hline Obligación 1 & Obligación 10 & Obligación 2 & Obligación 5 & Obligación 17 \\
\hline Obligación 23 & Obligación 15 & Obligación 3 & Obligación 6 & Obligación 19 \\
\hline Obligación 24 & Obligación 22 & Obligación 4 & Obligación 18 & \\
\hline Obligación 25 & Obligación 31 & Obligación 7 & Obligación 20 & \\
\hline Obligación 26 & & Obligación 8 & Obligación 21 & \\
\hline Obligación 27 & & Obligación 9 & Obligación16 & \\
\hline Obligación 28 & & Obligación 11 & & \\
\hline Obligación 29 & & Obligación 12 & & \\
\hline Obligación 30 & & Obligación 13 & & \\
\hline & & Obligación 14 & & \\
\hline
\end{tabular}

Fuente. Elaboración propia. 


\subsubsection{Defina el grado de relevancia de cada nivel}

Se define de acuerdo con la relevancia que tiene cada una de las obligaciones específicas para el logro del componente. Para definir la importancia que tiene cada uno de los niveles al logro del objeto contractual, el contratista, según su estrategia de la ejecución, asigna un ponderador tanto a los componentes como a las obligaciones específicas. Una vez definidos los ponderadores, se discutirán con el contratante para que conjuntamente se acuerde el nivel de contribución al logro del objeto contractual.

En este orden de ideas, en el ejemplo se definió la contribución de cada uno de los niveles. Como se observa en la tabla 4, los componentes misionales tienen los mayores ponderadores, mientras que el componente funcional hace un aporte marginal al logro del objeto contractual, no obstante, la sumatoria de los ponderadores debe sumar el $100 \%$.

Tabla 4. Ponderadores componentes

\begin{tabular}{|l|c|}
\hline Componentes & Wi (\%) \\
\hline Administrativo & 3 \\
\hline Mantenimiento & 30 \\
\hline Desplazamiento & 30 \\
\hline Seguridad & 18 \\
\hline Cumplimiento de normas & 19 \\
\hline Total & 100 \\
\hline
\end{tabular}

Fuente. Elaboración propia.
Para cada una de las obligaciones específicas, se definió un ponderador acorde con su nivel de contribución al logro del componente al que pertenece. Al respecto, debe tenerse en cuenta que la relevancia de cada obligación está directamente relacionada con el nivel de contribución al logro del bien o servicio. Si bien se reconoce que el ponderador es subjetivo de acuerdo con el punto de vista de quien lo define, para minimizar esa subjetividad se debe generar una justificación que evidencie las razones por las que se considera su nivel de mayo o menor aporte.

En nuestro caso de estudio, no todas las obligaciones tienen el mismo nivel de relevancia en los componentes misionales. Sin embargo, para el componente funcional, las obligaciones sí tienen el mismo nivel de contribución, lo que es lógico si se considera que son apoyo al funcionamiento del contrato (Tabla 5). 
Tabla 5. Ponderadores obligaciones específicas por componente

\begin{tabular}{|c|c|c|c|c|c|}
\hline Administrativo & Wi (\%) & Seguridad & Wi (\%) & $\begin{array}{l}\text { Desplazamien- } \\
\text { to }\end{array}$ & Wi (\%) \\
\hline Obligación 1 & 11 & Obligación 5 & 20 & Obligación 2 & 15 \\
\hline Obligación 23 & 11 & Obligación 6 & 10 & Obligación 3 & 10 \\
\hline Obligación 24 & 11 & Obligación 18 & 20 & Obligación 4 & 10 \\
\hline Obligación 25 & 11 & Obligación 20 & 20 & Obligación 7 & 15 \\
\hline Obligación 26 & 11 & Obligación 21 & 15 & Obligación 8 & 10 \\
\hline Obligación 27 & 11 & Obligación 16 & 15 & Obligación 9 & 15 \\
\hline Obligación 28 & 1 & Subtotal & 100 & Obligación 11 & 10 \\
\hline Obligación 29 & 1 & & & Obligación 12 & 5 \\
\hline Obligación 30 & 1 & & & Obligación 13 & 5 \\
\hline \multirow[t]{2}{*}{ Subtotal } & 100 & & & Obligación 14 & 5 \\
\hline & & & & Subtotal & 100 \\
\hline Mantenimiento & Wi (\%) & Cumplimiento de normas & Wi (\%) & & \\
\hline Obligación 10 & 45 & Obligación 17 & 10 & & \\
\hline Obligación 15 & 20 & Obligación 19 & 90 & & \\
\hline Obligación 22 & 30 & Subtotal & 100 & & \\
\hline Obligación 31 & 5 & & & & \\
\hline Subtotal & 100 & & & & \\
\hline
\end{tabular}

Fuente. Elaboración propia.

\subsubsection{Cálculo del indicador sintético}

Es una medida de agregación de los cálculos parciales de contribución de los componentes al logro del objeto contractual que se determina mediante el indicador de avance al objeto contractual (IAC).

$$
I A C=\sum_{n=1}^{n=5}(a j w i+a j w i+\cdots+a j w i)(1)
$$

\section{Donde:}

aj $\operatorname{con} \mathrm{J}=1, \ldots, \mathrm{n}$, de modo que $\mathrm{n}$ es igual a 5 que corresponde al número de componentes en estudio y wi representa la relevancia de la j-ésima variable, es decir, el ponderador conforme a la calificación obtenida. Como se observa en la ecuación 1, cada razón aj se multiplica por su correspondiente relevancia wi.

Gráficamente, el indicador sintético se conceptualiza como se muestra en la figura 3. 
Figura 3. Conceptualización indicador sintético.

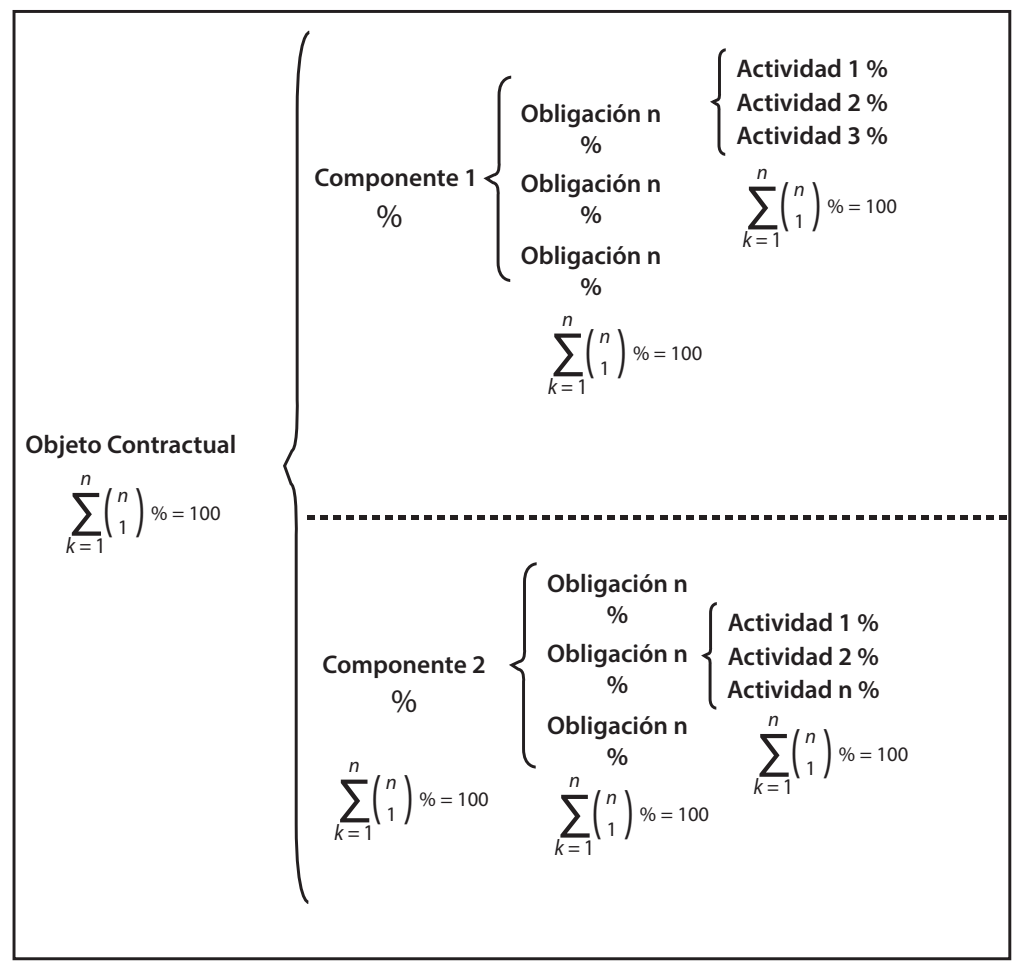

Fuente. Elaboración propia.

Mediante el IAC se cuenta con una nueva herramienta que permite hacer uso de las buenas características aportadas por las técnicas existentes, al tiempo que se contrarresten las desventajas o debilidades que presenta la atomización de obligaciones de un contrato. Para ello, a partir del seguimiento al cumplimiento de cada obligación, se obtiene un conjunto de indicadores simples I1, I2, In. Para poder hacer uso de estas observaciones de la forma más óptima posible, en un primer paso tendremos que convertir todos nuestros indicadores simples en indicadores del tipo "parcial», esto es, agregaremos de manera ponderada las calificaciones de avance según el componente. Una vez realizadas estas consideraciones, se calcula el nuevo indicador sintético. 


\section{Resultados}

Como figura del funcionamiento general del IAC, se presenta una aplicación práctica sobre un conjunto de datos referidos al análisis de avance de un contrato suscrito entre una entidad del Estado y un contratista, que arrojó los resultados que se observan en la figura 4.

Figura 4. Funcionamiento del IAC.
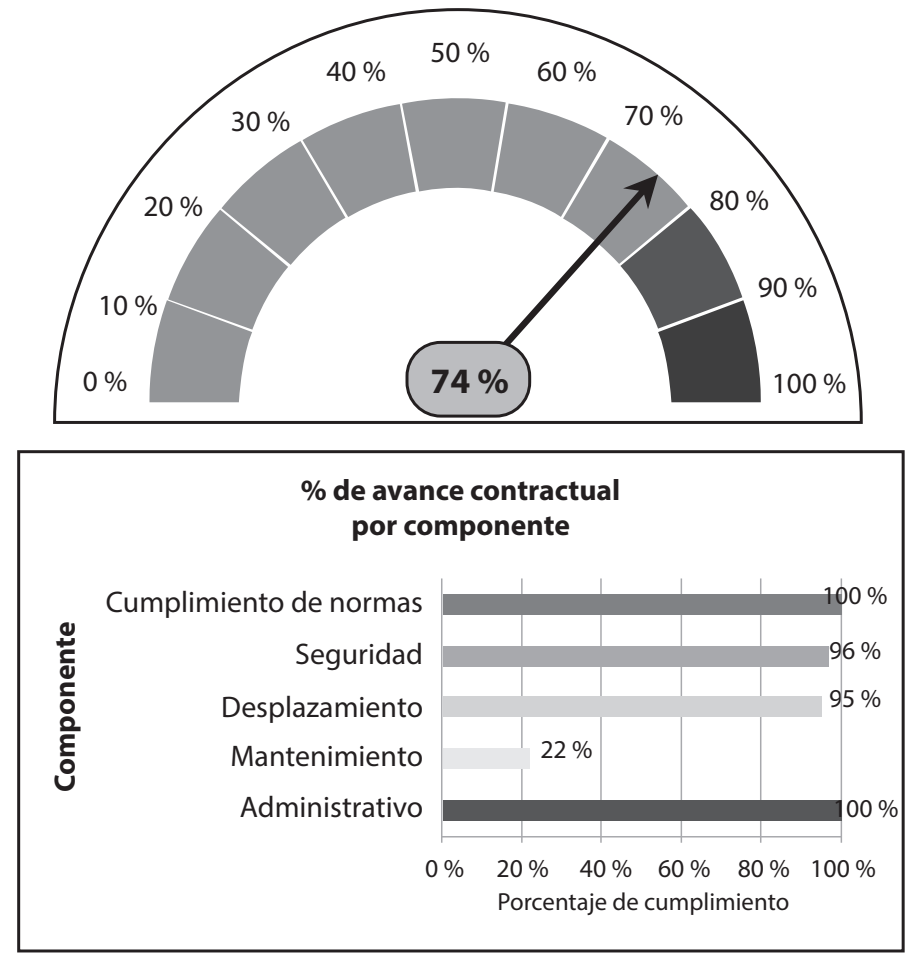

Fuente. Elaboración propia.

En concreto, hemos considerado 31 simples que recogen aspectos del nivel de avance de cada una de las obligaciones específicas del contratista. El objetivo es agregar la información proporcionada por el sistema de indicadores simples para calcular unos índices parciales. Para el cálculo, se utiliza la metodología basada en el análisis en componentes principales, ya que aquel se hace de forma objetiva y la información que se utiliza posee una base estadística que garantiza el análisis de información de los indicadores simples. Por tanto, analizaremos las correlaciones existentes entre los indicadores simples y los componentes principales calculados mediante los indicadores parciales (Tabla 6). 
Tabla 6. Correlaciones entre indicadores simples y componentes principales, mediante indicadores parciales.

\begin{tabular}{|c|c|c|c|c|c|c|}
\hline COMPONENTE & $\begin{array}{l}\text { Obligación } \\
\text { contractual }\end{array}$ & $\begin{array}{c}\text { (A) } \\
\text { Nivel de avance }\end{array}$ & $\begin{array}{c}\text { (B) } \\
\text { Ponderador }\end{array}$ & $\begin{array}{c}(C) \\
\text { Indicador } \\
\text { simpre }=\left(A^{*} B\right)\end{array}$ & $\begin{array}{l}\text { (E) } \\
\text { Ponderador de } \\
\text { componente }\end{array}$ & $\begin{array}{c}\text { Indicador } \\
\text { parcial }=\left(D^{*} E\right)\end{array}$ \\
\hline \multirow{9}{*}{ ADMINISTRATIVO } & 1 & $100 \%$ & $11,1 \%$ & $11,1 \%$ & \multirow{9}{*}{$3 \%$} & \multirow{9}{*}{$3,00 \%$} \\
\hline & 23 & $100 \%$ & $11,1 \%$ & $11,1 \%$ & & \\
\hline & 24 & $100 \%$ & $11,1 \%$ & $11,1 \%$ & & \\
\hline & 24 & $100 \%$ & $11,1 \%$ & $11,1 \%$ & & \\
\hline & 26 & $100 \%$ & $11,1 \%$ & $11,1 \%$ & & \\
\hline & 27 & $100 \%$ & $11,1 \%$ & $11,1 \%$ & & \\
\hline & 28 & $100 \%$ & $11,1 \%$ & $11,1 \%$ & & \\
\hline & 29 & $100 \%$ & $11,1 \%$ & $11,1 \%$ & & \\
\hline & 30 & $100 \%$ & $11,1 \%$ & $11,1 \%$ & & \\
\hline
\end{tabular}

(D) Agregado componente $=\sum_{n=1}^{n=9} 22,0 \%$

\begin{tabular}{|c|c|c|c|c|c|c|}
\hline COMPONENTE & $\begin{array}{l}\text { Obligación } \\
\text { contractual }\end{array}$ & $\begin{array}{c}\text { (A) } \\
\text { Nivel de avance }\end{array}$ & $\begin{array}{c}\text { (B) } \\
\text { Ponderador }\end{array}$ & $\begin{array}{c}(\mathrm{C}) \\
\text { Indicador } \\
\text { simple }=\left(\mathrm{A}^{*} \mathrm{~B}\right)\end{array}$ & $\begin{array}{l}\text { (E) } \\
\text { Ponderador de } \\
\text { componente }\end{array}$ & $\begin{array}{c}\text { Indicador } \\
\text { parcial }=\left(D^{*} E\right)\end{array}$ \\
\hline \multirow{4}{*}{ MANTENIMIENTO } & 10 & $18,9 \%$ & $45 \%$ & $8,5 \%$ & \multirow{4}{*}{$30 \%$} & \multirow{4}{*}{$6,6 \%$} \\
\hline & 15 & $38,5 \%$ & $20 \%$ & $7,7 \%$ & & \\
\hline & 22 & $8,4 \%$ & $30 \%$ & $2,5 \%$ & & \\
\hline & 31 & $66,4 \%$ & $5 \%$ & $3,3 \%$ & & \\
\hline
\end{tabular}

(D) Agregado componente $=\sum_{n=1}^{n=4} 22,0 \%$

\begin{tabular}{|c|c|c|c|c|c|c|}
\hline COMPONENTE & $\begin{array}{l}\text { Obligación } \\
\text { contractual }\end{array}$ & $\begin{array}{c}\text { (A) } \\
\text { Nivel de avance }\end{array}$ & $\begin{array}{c}\text { (B) } \\
\text { Ponderador }\end{array}$ & $\begin{array}{c}(\mathrm{C}) \\
\text { Indicador } \\
\text { simple }=\left(\mathrm{A}^{*} \mathrm{~B}\right)\end{array}$ & $\begin{array}{l}\text { (E) } \\
\text { Ponderador de } \\
\text { componente }\end{array}$ & $\begin{array}{c}\text { Indicador } \\
\text { parcial }=\left(D^{*} E\right)\end{array}$ \\
\hline \multirow{6}{*}{ SEGURIDAD } & 5 & $99 \%$ & $20 \%$ & $20 \%$ & \multirow{6}{*}{$18 \%$} & \multirow{6}{*}{$17 \%$} \\
\hline & 6 & $100 \%$ & $10 \%$ & $10 \%$ & & \\
\hline & 18 & $94 \%$ & $20 \%$ & $19 \%$ & & \\
\hline & 20 & $97 \%$ & $20 \%$ & $19 \%$ & & \\
\hline & 21 & $92 \%$ & $15 \%$ & $14 \%$ & & \\
\hline & 16 & $98 \%$ & $15 \%$ & $15 \%$ & & \\
\hline
\end{tabular}

(D) Agregado componente $=\sum_{n=1}^{n=6} 96,5 \%$ 
Tabla 6. Correlaciones entre indicadores simples y componentes principales, mediante indicadores parciales (Continuación)

\begin{tabular}{|c|c|c|c|c|c|c|}
\hline COMPONENTE & $\begin{array}{c}\text { Obligación } \\
\text { contractual }\end{array}$ & $\begin{array}{c}(\mathrm{A}) \\
\text { Nivel de avance }\end{array}$ & $\begin{array}{c}(\mathrm{B}) \\
\text { Ponderador }\end{array}$ & $\begin{array}{c}(\mathrm{C}) \\
\text { Indicador } \\
\text { simple }=\left(\mathrm{A}^{*} \mathrm{~B}\right)\end{array}$ & $\begin{array}{c}(\mathrm{E}) \\
\text { Ponderador de } \\
\text { componente }\end{array}$ & $\begin{array}{c}\text { Indicador } \\
\text { parcial }=(\mathrm{D} * \mathrm{E})\end{array}$ \\
\hline \multirow{2}{*}{$\begin{array}{c}\text { CUMPLIMIENTO } \\
\text { DE NORMAS }\end{array}$} & 17 & 1 & $10 \%$ & $10 \%$ & \multirow{2}{*}{$19 \%$} & $19 \%$ \\
\cline { 2 - 7 } & 19 & 1 & $90 \%$ & $90 \%$ & & $19 \%$ \\
\hline
\end{tabular}

(D) Agregado componente $=\sum_{n=1}^{\mathrm{n}=2} 100,0 \%$

\begin{tabular}{|c|c|c|c|c|c|c|}
\hline COMPONENTE & $\begin{array}{l}\text { Obligación } \\
\text { contractual }\end{array}$ & $\begin{array}{c}\text { (A) } \\
\text { Nivel de avance }\end{array}$ & $\begin{array}{c}\text { (B) } \\
\text { Ponderador }\end{array}$ & $\begin{array}{c}(\mathrm{C}) \\
\text { Indicador } \\
\text { simpre }=\left(A^{*} B\right)\end{array}$ & $\begin{array}{l}\text { (E) } \\
\text { Ponderador de } \\
\text { componente }\end{array}$ & $\begin{array}{c}\text { Indicador } \\
\text { parcial }=\left(D^{*} E\right)\end{array}$ \\
\hline \multirow{10}{*}{ DESPLAZAMIENTO } & 2 & $98 \%$ & $15 \%$ & $15 \%$ & \multirow{10}{*}{$30 \%$} & \multirow{10}{*}{$28,54 \%$} \\
\hline & 3 & $97 \%$ & $10 \%$ & $10 \%$ & & \\
\hline & 4 & $99 \%$ & $10 \%$ & $10 \%$ & & \\
\hline & 7 & $95 \%$ & $15 \%$ & $14 \%$ & & \\
\hline & 8 & $100 \%$ & $10 \%$ & $10 \%$ & & \\
\hline & 9 & $77 \%$ & $15 \%$ & $12 \%$ & & \\
\hline & 11 & $100 \%$ & $10 \%$ & $10 \%$ & & \\
\hline & 12 & $100 \%$ & $5 \%$ & $5 \%$ & & \\
\hline & 13 & $100 \%$ & $5 \%$ & $5 \%$ & & \\
\hline & 14 & $100 \%$ & $5 \%$ & $5 \%$ & & \\
\hline
\end{tabular}

(D) Agregado componente $=\sum_{n=1}^{\mathrm{n}=10} 95,1 \%$

Fuente. Elaboración propia.

Indicador sintético

$$
\mathrm{IAC}=\sum_{\mathrm{n}=1}^{\mathrm{n}=5}(3 \%+6,6 \%+28,54 \%+17 \%+19 \%)
$$

$\mathrm{IAC}=74,14 \%$ 


\section{Conclusiones}

La aplicación del Mosac es una herramienta que incrementa el éxito en la interventoría, dado que:

1) Identifica y clasifica el tipo de obligaciones y le permite al interventor focalizar sus puntos de atención.

2) Pondera las distintas obligaciones $y$ permite comprender la contribución de cada obligación al logro del objeto contractual.

3) Diseña los indicadores simples y parciales $\mathrm{y}$ hace posible un entendimiento del modelo lógico de interrelación de las obligaciones y su incidencia en el logro del objeto contractual.

4) Cuenta con un modelo cuantitativo para medir el avance al objeto contractual y posibilita a los interventores incrementar su efectividad en el seguimiento, lo que:

a) Garantiza el logro de bienes y servicios de calidad y contribuye con el mejoramiento del nivel de calidad de vida de la sociedad.

b) Asegura el cumplimiento de las condiciones pactadas. c) Cumple con lo exigido en los artículos 83 y 84 de la Ley 1474/2011, de 12 de julio:

a) Certificar como recibida a satisfacción obra que ha sido ejecutada a cabalidad.

b) Informar a la entidad contratante los hechos o circunstancias que puedan constituir actos de corrupción tipificados como conductas punibles, o que puedan poner o pongan en riesgo el cumplimiento del contrato, o cuando se presente el incumplimiento.

5) Cuantifica de manera objetiva el avance del objeto contractual, porque:

a) Genera recomendaciones a los contratistas y alertas a la entidad contratante.

b) Cuenta con información para la toma de decisiones que conminen al contratista al cumplimiento de lo contratado.

b) Toma cursos de acción que permiten cambiar la estrategia de ejecución, con el fin de cumplir con lo contratado. 


\section{Referencias}

Arriagada, R. (2002). Diseño de un sistema de medición de desempeño para evaluar la gestión municipal: una propuesta metodológica. Santiago de Chile: Instituto Latinoamericano y del Caribe de Planificación Económica y Social.

Baccarini, D. y Collins, A. (2003). Critical success factors for projects. Ponencia presentada en Proceedings of the 17th ANZAM Conference, Fremantle, Western Australia.

Balcázar Moreno, A. B. (2012). La supervisión de los contratos estatales en las entidades autónomas del nivel nacional de la administración pública (Tesis de maestría, Universidad del Rosario, Bogotá, Colombia).

Banco Interamericano de Desarrollo (1997). Evaluación: una herramienta de gestión para mejorar el desempeño de los proyectos. Recuperado de http://www.cedet.edu. ar/Archivos/Bibliotecas Archivos/72BID-OVE\%20 -\%20Marco\%20L\%C3\%B3gico.pdf

Colombia Compra Eficiente. (2016, diciembre 23). Supervisión einterventoría en contratos estatales. Recuperado de https://sintesis.colombiacompra.gov.co/content/ supervisión-e-interventoría-en-contratos-estatales

Concepto 1642/2005, de 9 de junio, trámite de la contratación estatal.

Echebarría, K. y Mendoza, X. (1999). La especificidad de la gestión pública: el concepto de management público. En C. Losada i Marrodán (ed.), ¿De burócratas a gerentes? Las ciencias de la gestión aplicadas a la administración del Estado (pp. 15-46). Washington, D. C.: Banco Interamericano de Desarrollo.

Guardia Girós, P. (2005). Estudio sobre la influencia de la deformación del indentador de interpretación de los ensayos de indentación instrumentada (Tesis de grado, Universitat Politècnica de Catalunya, Barcelona, España). Recuperado de https://upcommons.upc. edu/bitstream/handle/2099.1/3187/41781-1.pdf?sequence $=1$ \&isAllowed $=\mathrm{y}$

Ittner, C. y Larcker, D. (2000). Non-financial performance measures: What works and what doesn't. Financial Times, 16(10).
Ley 80/1993, de 28 de octubre, por la cual se expide el Estatuto General de Contratación de la Administración Pública.

Ley 1474/2011, de 12 de julio, por la cual se dictan normas orientadas a fortalecer los mecanismos de prevención, investigación y sanción de actos de corrupción y la efectividad del control de la gestión pública.

Mejía Lira, J. (2005). La evaluación como herramienta para una gestión pública orientada a resultados: la práctica de la evaluación en el ámbito público mexicano. Ponencia presentada en el X Congreso Internacional del CLAD sobre la reforma del Estado y de la administración pública, Santiago de Chile.

Metcalfe, L. (1999). La gestión pública: de la imitación a la innovación. En C. Losada i Madorrán (ed.), ¿De burócratas a gerentes? Las ciencias de la gestión aplicadas a la administración del Estado (pp. 47-86). Washington, D. C.: Banco Interamericano de Desarrollo.

Ortegón, E., Pacheco, J. F. y Prieto, A. (2005). Metodología del marco lógico para la planificación, el seguimiento y la evaluación de proyectos y programas. Serie Manuales, 42. Recuperado de https://repositorio.cepal.org/bitstream/ handle/11362/5607/S057518_es.pdf

Performance Management Methodology. (1998). Guide to a balanced scorecard performance management methodology. Washington, D.C.: U.S. Dept. of Commerce, Recuperado de http://web.cjcu.edu.tw/ lcc/Courses/BalancedScorecardPerfAndMeth\%20Tutorial.pdf

Ronda Pupo, G. A. y Marcané Laserra, J. Á. (2004). De la estrategia a la dirección estratégica. Modelo de dirección estratégica integrada: acercamiento a la complementación de los niveles estratégico, táctico y operativo. Primera parte. Ciencias de la Información, 35(1), 3-18.

Sentencia 15666/2011, de 10 de marzo, licitación que culmina con la celebración del contrato de obra pública regida por un pliego de condiciones incompleto, confuso y ambiguo. 
